\title{
A New Vision for Fusion Energy Research: Fusion Rocket Engines for Planetary Defense
}

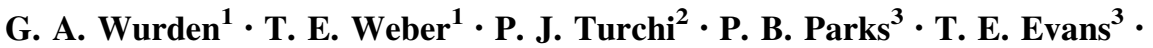 \\ S. A. Cohen ${ }^{4}$ J. T. Cassibry ${ }^{5}$ E. M. Campbell ${ }^{6}$
}

Published online: 16 November 2015

(c) The Author(s) 2015. This article is published with open access at Springerlink.com

\begin{abstract}
We argue that it is essential for the fusion energy program to identify an imagination-capturing critical mission by developing a unique product which could command the marketplace. We lay out the logic that this product is a fusion rocket engine, to enable a rapid response capable of deflecting an incoming comet, to prevent its impact on the planet Earth, in defense of our population, infrastructure, and civilization. As a side benefit, deep space solar system exploration, with greater speed and orders-of-magnitude greater payload mass would also be possible.
\end{abstract}

Keywords Fusion research - Fusion rocket engine . Comet deflection $\cdot$ Planetary defense $\cdot$ Nuclear explosive

The US Department of Energy's magnetic fusion research program, based in its Office of Science, focuses on plasma and fusion science [1] to support the long term goal of environmentally friendly, socially acceptable, and economically viable electricity production from fusion reactors [2]. For several decades the US magnetic fusion program has had to deal with a lack of urgency towards and inconsistent funding for this ambitious goal. In many American circles, fusion isn't even at the table [3] when it

G. A. Wurden

wurden@lanl.gov

Los Alamos National Laboratory, Los Alamos, NM, USA

Santa Fe, NM, USA

3 General Atomics, San Diego, CA, USA

4 Princeton Plasma Physics Laboratory, Princeton, NJ, USA

5 University of Alabama, Huntsville, Huntsville, AL, USA

6 Sandia National Laboratory, Albuquerque, NM, USA comes to discussing future energy production. Is there another, more urgent, unique, and even more important application for fusion?

\section{Fusion's Unique Application}

As an on-board power source and thruster for fast propulsion in space [4], a fusion reactor would provide unparalleled performance (high specific impulse and high specific power) for a spacecraft. To begin this discussion, we need some rocket terminology. Specific impulse is defined as $I_{s p}=v_{e} / g$, where $g$ is the usual Earth's gravitational acceleration constant and $v_{e}$ is the rocket propellant's exhaust velocity. The rocket equation, $\mathrm{M}_{\mathrm{f}} / \mathrm{M}_{\mathrm{o}}=\exp$ $\left(-\Delta \mathrm{V} / v_{e}\right)$, allows us to relate the final mass $\mathrm{M}_{\mathrm{f}}$ of the rocket divided by its initial mass $\mathrm{M}_{\mathrm{o}}$, to the change in velocity $\Delta V$ that it is capable of achieving. The rocket requires a power source with an output power $\mathrm{P}=\alpha \mathrm{M}_{\mathrm{s}}$, where we define $\alpha$ to be the specific power $\left(\mathrm{W} / \mathrm{kg}\right.$ ), and $\mathrm{M}_{\mathrm{s}}$ as the mass of the power supply (including the power conditioning, structures, and any waste heat radiators).

Today's best chemical rockets produce propellant exhaust velocities $\left(v_{e}\right)$ up to $4.5 \mathrm{~km} / \mathrm{s}$. Fission (nuclear thermal) rocket engines [5] could roughly double that, to about $8.5 \mathrm{~km} / \mathrm{s} \quad(<1 / 2 \mathrm{eV} / \mathrm{amu}$ temperature equivalent), constrained by material limits [6]. Electrically driven thrusters [7] are already quite efficient and have higher propellant exhaust velocities (corresponding to $\sim 5 \mathrm{eV} /$ $\mathrm{amu}$ ) but are usually limited in power resulting in low thrust, and are driven by limited electrical power/energy sources (photovoltaic or radioisotope). Development of high power, high thrust plasma thrusters has not been a 
priority, due primarily to a lack of mission need and the availability of adequate power sources in space.

In contrast, a working nuclear fusion core producing thrust through direct exhaust of hot plasma, could readily generate up to $\sim 1000 \mathrm{~km} / \mathrm{s}$ exhaust velocities, corresponding to $\sim 10 \mathrm{keV} / \mathrm{amu}$ [8], several hundred times higher exhaust velocities than today's high power chemical and nuclear thermal rocket engines. Lesser exhaust velocities could in principle be obtained as needed, through the co-expansion of additional cold propellant, to increase the propellant mass and optimize the exhaust velocity for individual mission requirements [9]. Importantly, the power available could be of order 100's of megawatts, and possibly higher. In all cases, one has to consider tradeoffs between specific impulse, thrust, power, cost and mission requirements for any comparison between different thruster approaches [10].

The metric for a fusion-powered rocket, as opposed to fusion-generated electricity, is based on performance per unit mass, rather than cents/kilowatt-hour. There are many pre-conceptual point designs for fusion rocket cores, ranging from generic fusion rocket systems studies [11, 12], to levitated dipoles [13], to mirror machines [14], to field reversed configurations [15], and magnetized target fusion [8, 16]. Even ST tokamaks [17] and laser fusion sources [18] have been suggested (although present incarnations aren't reactors, and even so, are much too massive). At the highest level, the unresolved research problem is that we need a working, compact, high thrust-to-mass ratio fusion core. It is clear that the present magnetic fusion approach, involving large tokamaks, i.e., ITER-like, are not matched to the needs for spacecraft propulsion. Alternative fusion reactor designs, more compact and far less massive, will be required. The 2003 Report to FESAC on "Nonelectric Applications of Fusion" [4] clearly recognized this application and that a fusion core for spacecraft propulsion might look different than today's mainline fusion approaches. Some requirements would be more stringent and some more relaxed. For example, with a rocket, the thrustto-mass ratio is a critical parameter; hence having a high- $\beta$ plasma ( $\beta$ is the ratio of the plasma kinetic energy density to magnetic energy density) is extremely important to reduce the mass of the magnets and their power supplies. Tritium could be carried on short missions, so a breeding blanket is not even needed. First wall requirements could be relaxed (good vacuum in space, and plasma-wall interactions less important for short duration missions). Furthermore, low neutron emissions are desirable to reduce the shielding and waste heat radiator mass, thereby improving specific power of the system, meaning that advanced fuels (such as D-He ${ }^{3}$ ) would be preferred over DT.

\section{Why Does Our Civilization Need Faster Spacecraft? Planetary Defense!}

Most prior works [8-18] considering the need for fusion rocket engines have focused on manned exploration of the solar system, the large distances involved, and in particular, for the need to get people to Mars [19] or Jupiter [20] quickly enough so that health risks for crews are minimized during long duration missions. However as numerous events have shown, from the Cretaceous-tertiary extinction to the recent meteorite explosion over Chelyabinsk, the solar system can be a dangerous place. Given this history and the potential for large impacts there is the clear need to be able to quickly protect the Earth from an incoming comet or asteroid by altering the intruder's orbit. Ways of doing this depend in part on how far out in time the object is identified, and are surveyed in 2004 NASA [21] and 2010 NRC (NAS) reports [22]. For decades of warning, (which may be the case for most discovered asteroids with three to seven year orbits), one can develop different deflection techniques than for cases with only months of warning. Fusion engines deployed on the surface of an asteroid have already been suggested for multi-month deflection of asteroids [23]. Unfortunately, for long-period or hyperbolic orbit objects, the $<1$-year warning scenario is more likely the case. Comets typically become detectable to telescopes at Mars to Jupiter distances as they approach the Sun [3-7 astronomical units (AU)]. Further, newly discovered comets with this type of orbit would also have extremely high closing velocities (in the $40-80 \mathrm{~km} / \mathrm{s}$ range, significantly faster than asteroids), with the closing speed depending on what component of the Earth's orbital vector of $30 \mathrm{~km} / \mathrm{s}$ adds or subtracts in a potential impact [24]. Plausible scenarios can be envisioned where we have only 6-18 months of warning time. Compared to asteroids in relatively short-period orbits, which in principle can be seen years in advance, comets are the infrequent but highly destructive threat requiring rapid response.

An example of a comet threat occurred in 2014, in addition to the better-known far-smaller Chelyabinsk meteor $\left(20 \mathrm{~km} / \mathrm{s}, 1.2 \times 10^{4}\right.$ metric Tons, 500 kilotons impact energy) in 2013 mentioned above. The first comet discovered in 2013, just after New Years on January 3, was detected from the Sidings Springs Observatory in the Southern Hemisphere [25]. It was named C/2013 A1 (at $56 \mathrm{~km} / \mathrm{s}, 0.7 \mathrm{~km}$ in diameter, weighing $3 \times 10^{8}$ metric Tons, it had 4 billion megatons of kinetic energy). For nearly 3 months after first being spotted, the best determination of the orbital elements did not rule out an impact on Mars, on October 19, 2014. In fact it missed Mars by only $1 / 3$ the Earth-Moon distance, at $140,000 \mathrm{~km}$ (see Fig. 1 below). Had it impacted, the blast would be visible 


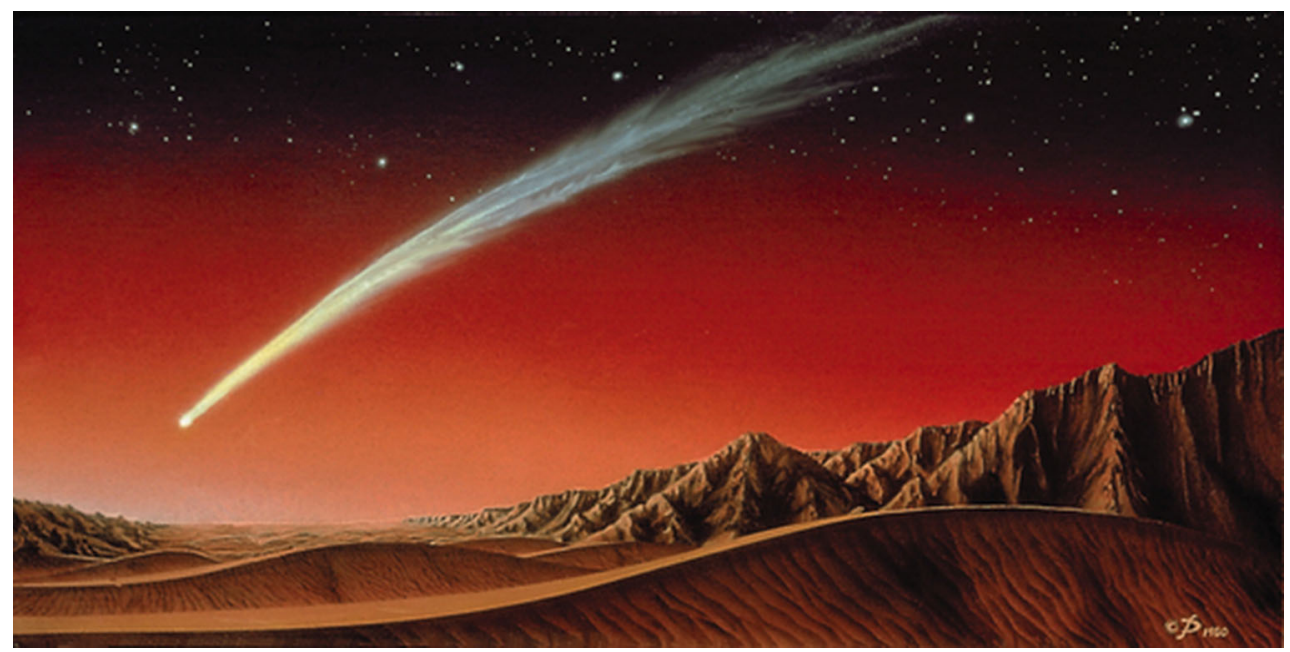

Fig. 1 Space artist Kim Poor's impression of a comet flyby of Mars. Comet C/2013-A1 passed extremely close to Mars on October 19, 2014

in the daylight from Earth and we probably would have lost all of our spacecraft on the ground and in orbit around Mars. In this example, even with an immediate launch for an intercept (after reasonable orbit determination), one would need a transit time for the intercept of $<6$ months.

From a threat analysis point of view, we are talking about defending against low probability, high consequence events. A reasonable question to ask, is how much money and effort should we be spending to make such a defense? Though smaller impacts are far more likely than large ones (20 m diameter once every 100 years, $1 \mathrm{~km}$ diameter once every million years) [26], the impact of a $1 \mathrm{~km}$ object (or larger) is capable of destroying our civilization. Indeed, such events with planetary wide consequences have occurred in the Earth's past. If one takes an actuarial approach, looking at damage amortized per year, versus probability of the damage occurring, there is a maximum/most probable worst case. We can see this illustrated as a sketch in Fig. 2. Here we assign a value of $\$ 1 \mathrm{M}$ of infrastructure per person, with a world population of $10^{10}$ people, with a frequency of $1 \mathrm{~km}$ impactors estimated at $10^{-6} /$ year. Small impacts can destroy a city, but not a continent. Intermediate impacts in the oceans can generate tsunamis, which could destroy multiple cities far removed from the impact at one time. Bigger impacts generate huge amounts of debris, firestorms, and planetary wide darkening. From a very simplistic linear viewpoint, this would imply that a planetary defense effort "insurance policy premium" of order $\$ 10 \mathrm{~B} /$ year would be appropriate. We are nowhere close to doing this now.

\section{The Nuclear Option}

To change a comet (or an asteroid) trajectory, one has to impart momentum to it. The further away from Earth that

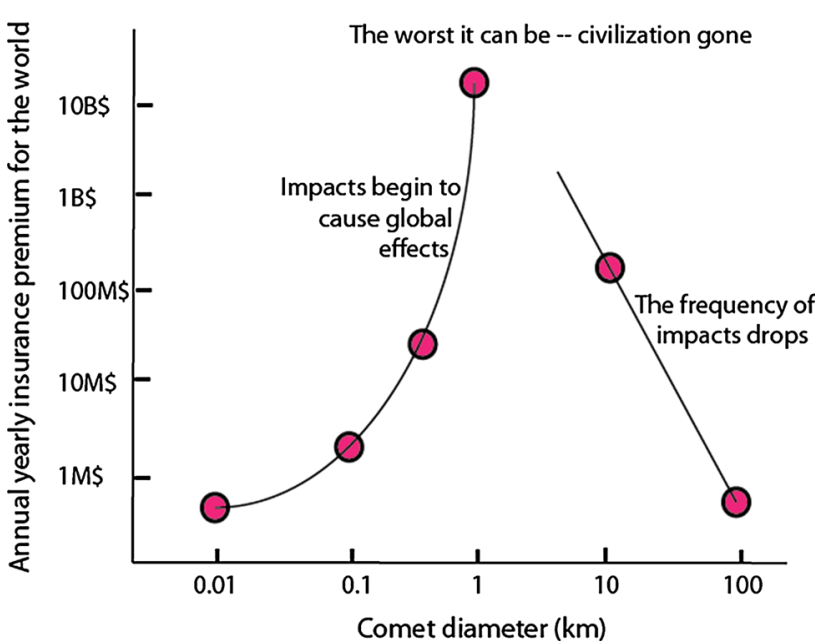

Fig. 2 What is it worth spending on Planetary Defense, from an actuarial point of view?

you can impart the momentum (i.e. the sooner in time), the smaller the required momentum change. For a comet, we wouldn't have much time to make the needed momentum change. One way to impart a significant momentum change quickly is to set off a nuclear explosion very close to the object ( $\leq 1 \mathrm{~km}$ distance) [27]. The ablation (caused by energy from the explosion being absorbed by the object) of one side of the surface of the object will cause a resulting "rocket" effect, and the thrust will change the object's trajectory. A 2007 NASA Report to Congress on "NearEarth Object Survey and Deflection Analysis of Alternatives" [28] concluded that a nuclear detonation near to an asteroid or comet is the best way to achieve the required deflection, especially in the case of a large object, and limited response times. In the 2004 NASA report [21], "nuclear deflection" (not nuclear fragmentation) is defined 
as using an intense radiation burst to cause sudden heating of the surface of the object, which ablates off material from the object's surface, resulting in a rocket effect. One would consider using the largest nuclear explosives ever tested (10-50 Megatons), consistent with getting them into deep space, and setting them off within $1 \mathrm{~km}$ distance of the target object. Rotation of the object (a show-stopper for some approaches) is inconsequential for this technique, and the relatively uniform surface irradiation might still enable deflection of objects with low structural integrity. Importantly, two international space treaties would have to be modified, first to allow nuclear explosives in orbit (as a launch vehicle is assembled), and then to permit nuclear explosions for Planetary Defense in deep space (far beyond the Earth).

\section{A Rapid Response Capability is Needed}

Because mission duration would be of the essence for a comet intercept mission, you don't have the velocity change $(\Delta V)$ budget, nor the time to waste to slow down and rendezvous with an incoming comet (which would require roughly $3 \times$ the intercept $\Delta V$ of a flyby). The comet should be hit on the fly. In particular, our analysis will focus on the shortest time duration, longest range encounter possible (to maximize the time for deflection effects to develop), which is the high-speed flyby case. But you have to get the nuclear explosive there in time to do any good. Consider that with our most powerful chemical rocket ever built-a Saturn V sitting on the pad (Fig. 3) - it takes 3-days to get out to lunar distances (Apollo going to the
Moon). If you want to send even a small payload to Mars distances, it takes 9-12 months (minimum energy Hohmann transfer trajectory) with conventional rocket engines. The future NASA Space Launch Systems (SLS) heavy launch booster will have similar capability as the former Saturn V rocket.

\section{Nuclear Rocket Engines}

As mentioned earlier, Nuclear Thermal (NT) rocket technology [29-31] increases the exhaust velocity from a limit of $\sim 4.5 \mathrm{~km} / \mathrm{s}$ for chemical rockets to $\sim 8.5 \mathrm{~km} / \mathrm{s}$, which is still far below what we need for the comet intercept mission. Los Alamos Scientific Laboratory (LASL) built more than a dozen such nuclear thermal rocket engines from 1955 to 1972, with Project Rover. Those engines used the most efficient propellant (hydrogen), and exhausted the hot gas at $<3000{ }^{\circ} \mathrm{K}$, limited by the temperature that the graphite reactor components could withstand. Aerojet and Westinghouse made a final design, of a J2 (third-stage Saturn V) engine replacement (Fig. 4), called NERVA [31], with about a factor of two higher specific impulse, $\mathrm{I}_{\mathrm{sp}}=900 \mathrm{~s}$, than the conventional chemical rocket engine it would replace.

Project Rover/NERVA was terminated in late 1972, because we weren't going to Mars with astronauts any time soon, and people were afraid of the risks of nuclear material being spread around the launch pad or into the Atlantic Ocean in case of an accident. Even so, for the high-value Planetary Defense missions, a rocket equipped with nuclear fission thermal engines might be useful for the

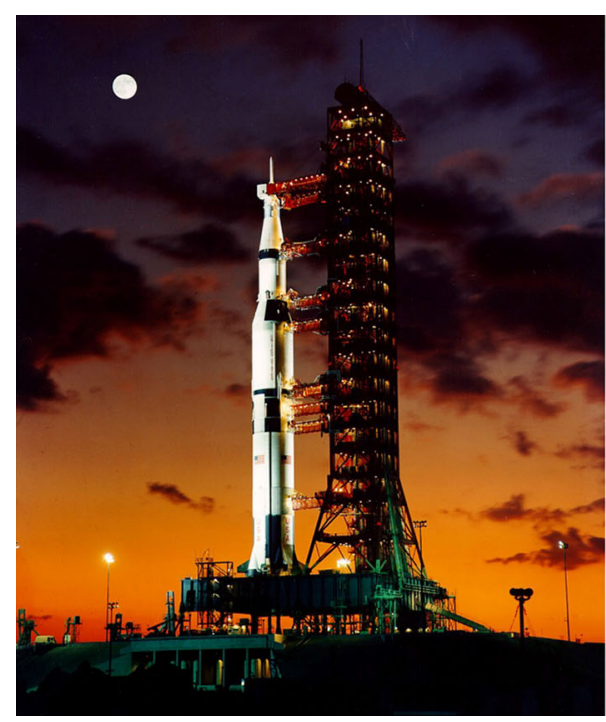

Fig. 3 (Credit NASA). Examples of the best heavy lift chemical rockets: Saturn V launch vehicle sitting on the pad (left), with third stage $\mathrm{J} 2$ engine specific impulse of $420 \mathrm{~s}$, and thrust of $1000 \mathrm{kN}$. New
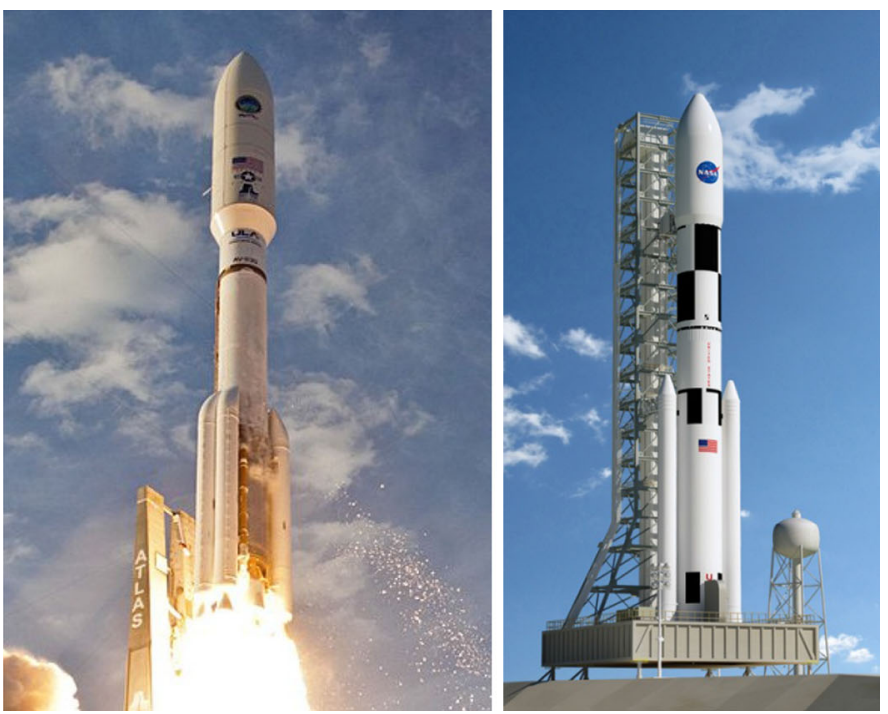

Horizons Pluto mission at launch on an Atlas V rocket (middle). NASA's new Space Launch Systems heavy launch vehicle (right) 

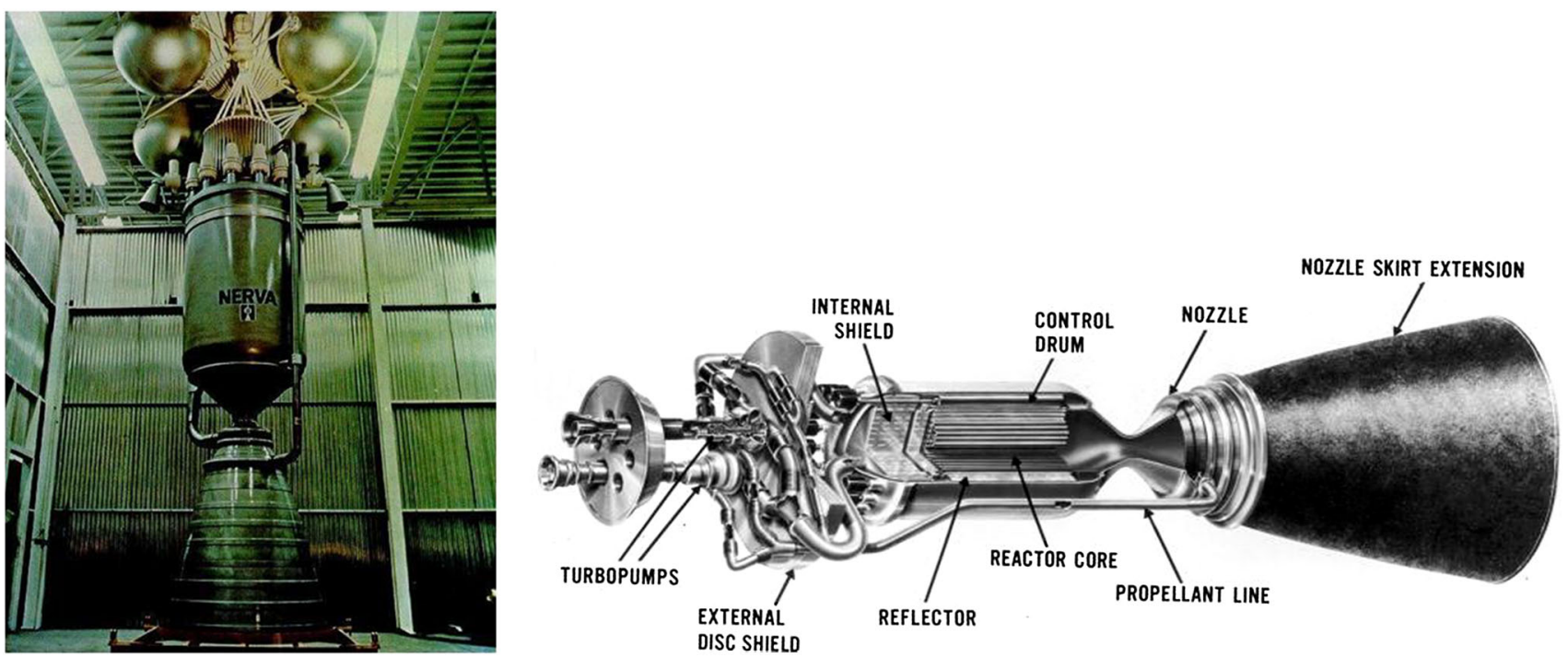

Fig. 4 (Credit: Atomic Energy Commission) The NERVA rocket engine, a nuclear thermal third stage replacement for the Saturn V with twice the $I_{s p}$ of the $\mathrm{J} 2$ engine. It was never used in space

asteroid threat, because it can get you out there $\sim 2 \times$ faster than with a chemical rocket.

Moving to plasma propulsion can even more significantly reduce the required propellant mass. As is well known from consideration of the rocket equation, there are diminishing returns for propellant mass savings when $v_{e} \gg$ $\Delta V$. At this point, propellant mass no longer dominates the calculus of mission space, and the spacecraft powerplant mass (with appropriate waste heat radiators) begins to dominate the total mission mass. Plasma propulsion could be via nuclear fission electrically powered thrusters (nuclear electric propulsion-NEP), fusion electric propulsion (FEP), or eventually fusion core exhaust (fusion propulsion-FP). In a planetary defense scenario, time is of the essence. Thus, traditional low-thrust plasma propulsion schemes (such as Hall or electrostatic ion thrusters) cannot be used. Even experimental plasma thruster concepts [6, 32] could only be considered if powered with a high specific power $(\alpha)$ source $(>1-10 \mathrm{~kW} / \mathrm{kg})$.

The problem with typical solar or even nuclear electric propulsion (EP) techniques is that the specific power $\alpha$ of the energy source is much too low unless we use a fusion core with advanced (low neutron output) fuels. Controlled fusion is the only potential energy source, other than nuclear explosives or antimatter, with the required specific power. While solar-electric systems have a specific power (at the Earth distance from the Sun) of order $\sim 100 \mathrm{~W} / \mathrm{kg}$, that decreases by a factor of $1 / 25$ at Jupiter, and $1 / 100$ at Saturn distances. Taking a fission example, the SAFE-400 nuclear Brayton cycle reactor and radiator system [33] would produce $100 \mathrm{~kW}$ electric power, with a mass of $584 \mathrm{~kg}$, for a specific power (without a thruster) of $171 \mathrm{~W} /$ kg. Coupling it to an $80 \%$ efficient ion thruster of the NSTAR [34] type presently in use on the Dawn mission [35], which has an specific impulse $\mathrm{I}_{\mathrm{sp}}=3100 \mathrm{~s}$ (but using 40 units, with a combined weight of $1000 \mathrm{~kg}$, corresponding to $100 \mathrm{~kW}$ of available electric energy), one would have a system specific power of $63(\mathrm{~W} / \mathrm{kg})$, but with a thrust of only $4 \mathrm{~N}$. By instead invoking an open-cycle (direct thrust) fusion rocket [9], rather than the typical nuclear-electric system for which the fission reactor waste heat has to be rejected, one could greatly improve the specific power while increasing the $\mathrm{I}_{\mathrm{sp}}$, and also greatly increase the total available thrust (total power).

\section{The Threat: Comets are More Troubling than Asteroids}

For long-lead time asteroid deflection missions (decade timescales), current technology has demonstrated the capability to intercept an object in the inner solar system (for example, the Ceres or Deep Impact missions). However we have not yet actually attempted deflections of asteroids with any technologies, nor compared such experiments to simulations. But comets pose a more difficult problem. They are generally large (civilization-ending, 1-10 km size), are inbound from any angle (out of the plane of the ecliptic), have high orbital speeds by the time they approach Earth, and will only provide short warning times, $\sim 1$ year, if we are looking.

Given the previous points, it is almost assured that a comet intercept mission will require spacecraft velocities of roughly of the comet's velocity to reach the comet in time to attempt a deflection (see Fig. 5). A long period 
Fig. 5 Approximate intercept trajectories for 3 and 6 month lead times. Even these examples require the interceptor to cover multiple-AU distances to gain the largest possible "lever arm"

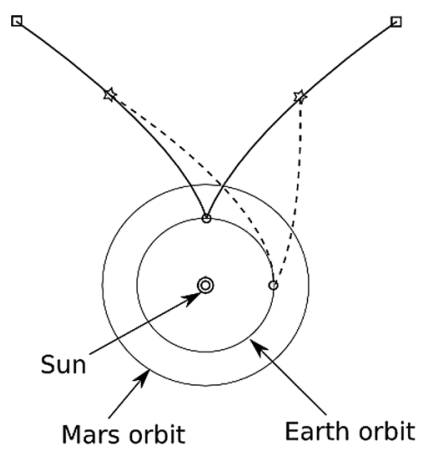

3-month lead-time

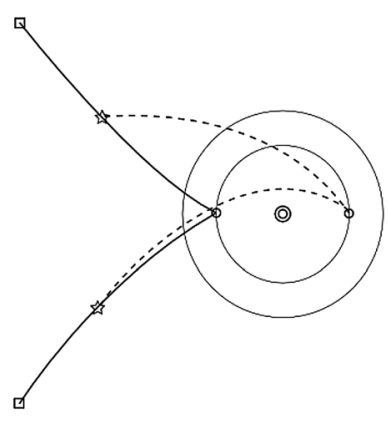

6-month lead-time $\square \quad$ Position of comet at time of spacecraft launch

Sosition of comet at time of intercept

Position of earth at time of spacecraft launch and time of intercept comet's speed as it approaches the Sun, is on the order of the solar system escape velocity $(42.1 \mathrm{~km} / \mathrm{s})$. Knowing that the Earth's orbital speed is $30 \mathrm{~km} / \mathrm{s}$ and that the Earth orbits at 1 astronomical unit $(1 \mathrm{AU}=149,000,000 \mathrm{~km})$ from the Sun, one quickly infers from this schematic diagram that an interceptor would need to cover distances of order 3-5 AU in a time less than the time to impact. Consider the 12-month warning case, and a comet discovered $10 \mathrm{AU}$ out from the Sun (at the distance of Saturn). Let's suppose we should intercept in $\sim 1 / 2$ of the time remaining to impact of the comet with Earth. The necessary $\Delta V$ is $\sim 5 \mathrm{AU} / 6$ months $=53 \mathrm{~km} / \mathrm{s}$. Just to do the flyby intercept requires a huge $\Delta V$. The fastest vehicle we have ever launched from the Earth, was the New Horizons spacecraft on its way to Pluto (with only a $465 \mathrm{~kg}$ payload @ $16.2 \mathrm{~km} / \mathrm{s}$, but a rocket weighing 570,000 kg at launch), which took only $9 \mathrm{~h}$ to pass the Moon's orbit after launching from Earth on an Atlas V511 rocket with Centaur and Star 48B upper stages (see Fig. 3). While the Pioneer, Voyager, and now New Horizons space probes have achieved solar system escape velocity, multiple planetary fly-bys were used to boost the spacecraft velocity, which requires proper planetary alignment (takes a long time and alignments rarely occur), and places the spacecraft in a very limited range of trajectories. Chemical rockets and gravity assists cannot be counted on for our proposed long-period comet intercept mission.

The induced comet deflection must have a sufficient effect, an Earth miss in distance, $\mathrm{R}_{\mathrm{m}}$, which can be predictable (beyond orbital element uncertainties) months in advance. For example, an Earth-Moon distance represents a "miss" of 400,000 km, whereas the Earth covers a distance in its orbit equal to the diameter of the Earth in $7 \mathrm{~min}$. By imparting a velocity change of $10 \mathrm{~m} / \mathrm{s}$ to the comet, the remaining time $\left(180\right.$ days $\left.=1.5 \times 10^{7} \mathrm{~s}\right)$, produces a miss distance of $\sim 150,000 \mathrm{~km}$, ignoring any closing speed effects of the Earth itself.

By specifying the distance at which the comet is detected and characterized as a threat to earth, and the deflection needed to miss the earth, we can determine the necessary exhaust speed and time, and thereby prescribe the necessary specific power for an intercept mission. We define $\tau$ as the time needed to attain the necessary velocity increment $\Delta V$ for the postulated mission. In fact $\tau$ might be as long as the mission duration. Naturally, the shorter the thrust duration, the higher the required specific power $\alpha$ [in watts $/ \mathrm{kg}$, including any conversion inefficiencies] required to achieve the necessary $\Delta V$. We take the initial mass of the space vehicle to be $\mathrm{M}_{0}$, so that $\mathrm{M}_{0}=\mathrm{Mp}+\mathrm{Ms}+\mathrm{M}_{\mathrm{L}}$, the propellant, power supply, and payload masses respectively. Through the rocket equation, the payload mass fraction, $\mathrm{M}_{\mathrm{L}} / \mathrm{M}_{\mathrm{o}}$ becomes,

$\left.\mathrm{M}_{\mathrm{L}} / \mathrm{M}_{\mathrm{o}}=\exp \left(-\Delta \mathrm{V} / v_{e}\right)-\mathrm{M}_{\mathrm{s}} / \mathrm{M}_{\mathrm{o}}\right)$

With $\mathrm{M}_{\mathrm{s}}=\mathrm{P} / \alpha=\dot{m} v_{e}^{2} / 2 \alpha$ for a propellant mass flow rate

$\dot{m}=\mathrm{M}_{\mathrm{o}}\left[1-\exp \left(-\Delta \mathrm{V} / v_{e}\right)\right] / \tau$

based on the duration of propellant exhaust $\tau$

So,

$\mathrm{M}_{\mathrm{L}} / \mathrm{M}_{\mathrm{o}}=\exp \left(-\Delta \mathrm{V} / v_{e}\right)-\left(v_{e}^{2} / 2 \alpha \tau\right)\left[1-\exp \left(-\Delta \mathrm{V} / v_{e}\right)\right]$

This nonlinear equation for $\mathrm{M}_{\mathrm{L}} / \mathrm{M}_{\mathrm{o}}$ in terms of $v_{e}$ and $\Delta \mathrm{V}$ is plotted in Stuhlinger [36], Ion Propulsion for Space Flight, chapter 4, p. 79, Figure 4.8, and provides the exhaust speed and velocity change in terms of $\alpha \tau$. Furthermore, to begin, we postulate that the payload mass fraction (for a nuclear explosive and tracking system) is nearly negligible compared to the vehicle mass. Then, again from Stuhlinger, in this limit of negligible payload mass, the necessary exhaust speed $v_{e}$ and velocity increment $\Delta V$ are then 0.5 and 0.81 times the characteristic speed $\sqrt{2 \alpha \tau}$, where again $\alpha$ is the specific power (W/kg) and $\tau$ is the time (s) required to attain the needed $\Delta V$. This shows us immediately the need to accomplish high specific power in order to enable a short-time mission. In actuality, one needs a finite useful payload mass. We suggest, for 
example, a 50 metric ton payload (nuclear explosive, reduced shielding, terminal tracking, final maneuvering) might be appropriate, since for a one-way flyby mission, our (unmanned) payload mass can be considerably smaller than the case of the previously postulated Discovery II manned mission to Jupiter [17].

With the needed $\Delta V$ upwards of $100 \mathrm{~km} / \mathrm{s}$ as indicated earlier, the exhaust speed would be $61 \mathrm{~km} / \mathrm{s}$ or a specific impulse of about $6300 \mathrm{~s}$. For the 180 day mission out to Saturn (distances), then we need a specific power of $500 \mathrm{~W} / \mathrm{kg}$, to deliver negligible useful payload... and more for a finite payload fraction. Even this combination is well beyond the range of fission nuclear thermal (NT) rockets, let alone nuclear electric (NEP) propulsion.

Finally, there is one other potential solution for the comet intercept mission. In the late 1950's, Ted Taylor and Freeman Dyson's DARPA “Project Orion” study proposed a spacecraft propelled using multiple pulsed nuclear denotations [37], (replete with unique virtues and vices), with a specific impulse of more than $6000 \mathrm{~s}$ and with high specific power. Stockpiling and launching thousands of nuclear explosives, for an indeterminate time, of course has a lot of other issues. It was revisited in the later Project Daedalus study from 1973 to 1978 by the British Interplanetary Society [38].

\section{Deflection Energy Requirements}

In more detail we now consider how the required deflection places demands on the nuclear explosive yield needed, and it's positioning with respect to the comet.

For lateral deflection $\mathrm{v}_{\mathrm{o}}$ in ideal, minimum energy, case, we would need an energy absorbed by the comet:

$\mathrm{W}_{\mathrm{o}}=\mathbf{M} \mathrm{v}_{\mathrm{o}}^{2} / 2$

where $\mathbf{M}$ is the comet mass and the deflection velocity $\mathrm{v}_{\mathrm{o}}$ is purely perpendicular to the comet's initial velocity V. A $1 \mathrm{~km}$ diameter comet, with density of $0.6 \mathrm{~g} / \mathrm{cm}^{3}$ has a mass of $3 \times 10^{11} \mathrm{~kg}$, and a deflection of $10 \mathrm{~m} / \mathrm{s}$ corresponds to an energy $\mathrm{W}_{0}=1.5 \times 10^{7} \mathrm{MJ}$.

Now if we consider (in the initial frame of motion of the comet) the ablated material to be treated as a rocket exhaust of average directed speed $\mathrm{v}$, and we expel a mass $\mathrm{m}$, then by momentum conservation:

$\mathbf{M v}_{\mathrm{o}}=\mathrm{mv}$

The total (directed) kinetic energy in the exhaust (not counting internal energy of the plume or radiation losses) becomes:

$\mathrm{mv}^{2} / 2=\mathbf{M v v}_{\mathrm{o}} / 2$

Compared to the minimum energy $\mathrm{W}_{\mathrm{o}}$, we therefore need more energy by a factor of $\mathrm{K}=\mathrm{v} / \mathrm{v}_{\mathrm{o}}$. For example, for our deflection $\mathrm{v}_{\mathrm{o}}=10 \mathrm{~m} / \mathrm{s}$ and an ablatant exhaust of $\mathrm{v}=4 \mathrm{~km} / \mathrm{s}$, the yield of the nuclear explosive must increase by $\mathrm{K}=400$. As an interesting side note, a spacecraft with a mass of 50 metric tons, closing at a speed of $100 \mathrm{~km} / \mathrm{s}$, carries a kinetic energy of $2.5 \times 10^{8} \mathrm{MJ}$, which is more than the energy $\mathrm{W}_{0}$ discussed above, but still much less than what the impulse energetics (and momentum conservation) requires to create this rocket plume from the comet surface.

\section{Equations for Long Range Encounter}

Continuing, we now need to relate the intercept time, the specific power $\alpha$, closing velocities and distances, to the deflection, earth miss distance, and required nuclear yield. Consider for the simple case of constant power and exhaust speed, then total mass flow out of the rocket can be used as a measure of time. Thus, the distance $x$ traveled in time $\tau$ is:

$\mathrm{x}=\int \mathrm{u} \mathrm{dt}=\int \mathrm{u}(-d M / \dot{M})$

where $\mathrm{M}$ is the instantaneous mass of the spacecraft which is diminished at a rate $\dot{M}(>0)$. The limits of integration are $\mathrm{M}_{\mathrm{o}}$ at time $\mathrm{t}=0$ and $\mathrm{M}=\mathrm{M}_{\mathrm{o}} \exp \left(-\mathrm{u} / v_{e}\right)$ at the time $\tau$ that the spacecraft has attained a speed $\mathrm{u}$ at constant exhaust speed $v_{e}$. The spacecraft speed is related to instantaneous mass and the exhaust speed by the rocket equation:

$\mathrm{u} / v_{e}=-\ln \left(M / M_{o}\right)$

The distance traveled is then:

$$
\begin{aligned}
\mathrm{x} & =v_{e}\left(M_{o} / \dot{M}\right) \int \ln \mathrm{M} / \mathrm{M}_{\mathrm{o}} \mathrm{d}\left(\mathrm{M} / \mathrm{M}_{\mathrm{o}}\right) \\
& =v_{e}\left(M_{o} / \dot{M}\right)\left[\mathrm{M} / \mathrm{M}_{\mathrm{o}}\left(\ln \left(\mathrm{M} / \mathrm{M}_{\mathrm{o}}\right)-\left(\mathrm{M} / \mathrm{M}_{\mathrm{o}}\right)\right]\right.
\end{aligned}
$$

where the limits of integration are 1 and $\exp \left(-\mathrm{u} / v_{e}\right)$. We therefore have

$\mathrm{x}=v_{e}\left(M_{o} / \dot{M}\right)\left\{1-\left[1+\left(\mathrm{u} / v_{e}\right)\right] \exp \left(-\mathrm{u} / v_{e}\right)\right\}$

$=v_{e} \tau\left\{1-\left[1+\left(\mathrm{u} / v_{e}\right)\right] \exp \left(-\mathrm{u} / v_{e}\right)\right\} /\left[1-\exp \left(-\mathrm{u} / v_{e}\right)\right]$

Using Stuhlingler's notation of velocities normalized to $(2 \alpha \tau)^{1 / 2}$, then for the limit of zero payload, we have normalized speeds $\mathrm{u}^{*}=0.81$ and $v_{e}^{*}=0.5$, we have $\mathrm{u}^{*} /$ $v_{e}^{*}=1.62$, and $\mathrm{x}=0.6 v_{e} \tau$. Note, although we don't do it here, if we let the payload ratio increase to 0.2 , then $\mathrm{u}^{*}=0.53$ and $v_{e}^{*}=0.707$, so $\mathrm{u}^{*} / v_{e}{ }^{*}=0.75$, and $\mathrm{x}=0.329 v_{e} \tau$ (Which would later change the coefficient in denominator of Eq. (12)). More simply put, if we want a higher payload mass fraction, the spacecraft will not get as far in the same time. 
With exhaust speed of the intercept vehicle $v_{e}=0.5$ $(2 \alpha \tau)^{1 / 2}$ and final delta- $\mathrm{V}$ of the vehicle, $\Delta \mathrm{V}=0.81(2 \alpha \tau)^{1 / 2}$, we have that the time for a flyby encounter (thrusting constantly all the way from the Earth) is:

$\tau=\frac{\left(\mathrm{R}_{\mathrm{D}}-\mathrm{R}_{\mathrm{o}}\right)}{\left[0.6 v_{e}+\left(\mathrm{V}+\mathrm{V}_{\mathrm{E}}\right)\right]}$

where $R_{D}$ and $R_{o}$ are the radius of detection and radius of earth's orbit, respectively, $\mathrm{V}$ is the comet speed (assumed to be constant, even though it gets faster as it approaches the Sun) and $V_{E}$ is the appropriate speed of the earth given the variation of angle during the comet's approach for an intercept time comparable to a quarter to half of the earth's period. The sum of $\mathrm{V}+\mathrm{V}_{\mathrm{E}}$ represents the comet's closing speed on the Earth. The average speed of the craft, for constant exhaust velocity and constant thrust, integrating from 0 to $\tau$, is the other term in the denominator.

Note that because the interceptor exhaust speed here is taken to be $v_{e}=0.5(2 \alpha \tau)^{1 / 2}$, the equation for intercept time could be solved (as a cubic equation) for a given specific power. However, it is more instructive to specify $v_{e}$ to obtain $\tau$ (under the time constraint of the incoming (undeflected) comet impact, which sets the maximum allowed $\tau$, while also needing to intercept at large fraction of $\mathrm{R}_{\mathrm{D}}$, to minimize the necessary deflection impulse) and then find the necessary value of $\alpha$. Our simplified estimate isn't meant to preclude a proper astrodynamics calculation for the comet and spacecraft, with curved trajectories, varying velocities, and possible out-of-the-equatorial plane considerations.

We take the solid angle represented by the comet at the intercept standoff distance $r_{i}$, the diameter of the comet $d_{c}$, and define the ablation efficiency factor $\varepsilon_{a b l}$ to include the fraction of the nuclear-explosive output in soft X-rays and the conversion of this output into the directed kinetic energy of the ablation-rocket exhaust. Since miss distance $\mathrm{R}_{\mathrm{m}}$ can be written in terms of a deflection angle with a lever arm distance mapped back to the time of intercept, we can relate that angle with the previous equations for the needed comet impulse [4-6] to write down the necessary nuclear yield:

$Y=\frac{16 \mathrm{r}_{\mathrm{i}}^{2}}{\mathrm{~d}_{\mathrm{c}}^{2}} \frac{K}{(0.5) \varepsilon_{\mathrm{abl}}}\left(\frac{4 \pi}{3} \frac{\rho_{c} d_{c}^{3}}{8} \frac{V^{2}}{2}\right) \frac{\mathrm{R}_{m}^{2}}{\left[\left(\mathrm{R}_{\mathrm{D}}-\mathrm{R}_{\mathrm{o}}\right)-\left(\mathrm{V}+\mathrm{V}_{\mathrm{E}}\right) \tau\right]^{2}}$

where we have the factor 0.5 in the denominator coming from averaging over the surface normal of the comet, $\rho_{c}$ is the comet mass density, $r_{i}$ is the intercept distance (centerto-center) and $d_{c}$ is the diameter of the comet. The distance by which the comet misses the earth, at its closest approach, is $\mathrm{R}_{\mathrm{m}}$. Yield goes as the square of the eventual miss distance, due to required ablation energy scaling as the square of the impulse deflection velocity.

If we eliminate $\tau$ in Eq. 13, using Eq. 12, then we obtain a more interesting form:

$$
Y=\frac{16 \mathrm{r}_{\mathrm{i}}^{2}}{\mathrm{~d}_{\mathrm{c}}^{2}} \frac{K}{(0.5) \varepsilon_{\mathrm{abl}}}\left(\frac{4 \pi}{3} \frac{\rho_{c} d_{c}^{3}}{8} \frac{V^{2}}{2}\right) \frac{\mathrm{R}_{m}^{2}}{\left(\mathrm{R}_{\mathrm{D}}-\mathrm{R}_{\mathrm{o}}\right)^{2}}\left[1+\frac{\left(\mathrm{V}+\mathrm{V}_{\mathrm{E}}\right)}{0.6 v_{e}}\right]^{2}
$$

The importance of achieving early detection (large $\mathrm{R}_{\mathrm{D}}$ ) of the comet, and a high exhaust velocity $v_{e}$ for the intercept vehicle is apparent. Clearly, the solution diverges if the detection distance approaches the Earth's orbital distance.

For the case of a desired $\mathrm{v}_{\mathrm{o}}=10 \mathrm{~m} / \mathrm{s}$, with an initial comet velocity $\mathrm{V}=25 \mathrm{~km} / \mathrm{s}$, then $\mathrm{K}=400$. With detection/characterization at $10 \mathrm{AU}$, an average earth speed component of $0.7 \times 30 \mathrm{~km} / \mathrm{s}=21 \mathrm{~km} / \mathrm{s}$, and an interceptor rocket exhaust speed of $200 \mathrm{~km} / \mathrm{s}$, we can intercept at about 4.9 AU in 93 days, but require a specific power $\alpha=6153 \mathrm{~W} / \mathrm{kg}$, which is well beyond anything except fusion. Using Eq. (14), we can estimate the required nuclear yield. To generate an Earth miss distance $\mathrm{R}_{\mathrm{m}}=40,000 \mathrm{~km}$, for a $1 \mathrm{~km}$ diameter comet with average density of $0.6 \mathrm{gm} / \mathrm{cm}^{3}$, with a $25 \%$ ablation efficiency of a single detonation, at a distance $r_{i}=1 \mathrm{~km}$, a yield of 20 Megatons is needed. Therefore while a single detonation of only 10 Megatons isn't quite enough, the biggest nuclear device ever tested on Earth (Russian, mass of 27 metric tons, $50 \mathrm{MT}$ yield) would have some margin. Furthermore, you need to make a big enough deflection to insure that the "miss distance" is measurable (beyond error bars, due to orbital parameter uncertainties, and short baselines for establishing the orbit parameters) in the time you have remaining before an impact. This would drive you towards even larger deflections, and therefore larger yields. More detailed calculations taking into account radiation and materials effects are clearly needed.

Even so, you would need multiple intercept vehicles and payloads, not only for redundancy, but to get sufficient deflection, while also having the ability to make corrections by iterating with multiple attempts. For a successful intercept and deflection, there are at least two more difficulties which come to mind: (1) Surviving the micrometeorites on approach, and (2) detonating a nuclear explosive package with sufficiently precise timing and targeting. Due to the high closing speeds (up to $\sim 100 \mathrm{~km} / \mathrm{s}$ ), a $10 \mathrm{~ms}$ triggering uncertainty corresponds to a $1 \mathrm{~km}$ error, which is about the desired standoff distance. 
Table 1 Energy sources routinely used to make electricity today (2010) [39]

\begin{tabular}{lllc}
\hline Primary energy source & Nominally $\mathrm{CO}_{2}$ free & Current capacity (\%) & Expected lifetime (years) \\
\hline Natural gas & No & & 100 \\
Coal & No & 80.6 & 400 \\
Oil & No & & $<50$ \\
Biomass & Neutral & 11.4 & $>400$ \\
Wind & Yes & 0.5 & $>1000$ \\
Solar photovoltaic & Yes & 0.06 & $>1000$ \\
Solar thermal & Yes & 0.17 & $>1000$ \\
Hydro & Yes & 3.3 & $>1000$ \\
Wave/tidal & Yes & 0.001 & $>1000$ \\
Geothermal & Yes & 0.12 & $>1000$ \\
Nuclear fission & Yes & 2.7 & $>400$ \\
\hline
\end{tabular}

\section{Back to the Future: Electricity Generation and/ or Rocket Propulsion?}

As a strategic goal for fusion energy research, clean $\mathrm{CO}_{2}$-free electricity generation does not offer a unique value proposition in the way that a fusion rocket for Planetary Defense does. There are at least 11 different ways to make electricity today, seven of which are "mostly $\mathrm{CO}_{2}$ free" (see Table 1). All of these techniques work today, with varying costs, societal acceptance, risk and differing abilities to meet future demands, and all of them are simpler than an as-yet-hypothetical fusion energy reactor. Among the existing " $\mathrm{CO}_{2}$ free" options, until cost effective large-scale storage is demonstrated, only nuclear fission has both the capability for future scalability for increased demand and steady output capability for base-load requirements. Nuclear fission of course has its many issues, including proliferation, safety and impact of accidents, spent fuel disposal and society acceptance. All of these impact the construction cost, which today in the developed world is excessive (overnight construction costs in excess of $\$ 5000 / \mathrm{kW}$ ) and prevents large scale deployment. There are several reactor designs and concepts that can address these concerns but they are not being actively pursued today. Given the scientific immaturity (we have yet to produce net fusion energy gain), the technical and engineering complexity envisioned for fusion power plants, the existence of well-established means of producing electricity whose cost of construction, operations, and the net cost of electricity (cents/kW-h) is well known, and the risk adverse nature of public utilities and the energy industries, the lack of enthusiasm for electricity produced by fusion can be readily understood.

Instead of electricity, we have identified a goal for fusion research in this paper, which satisfies a need that is not provided by any other existing product. Society is beginning to understand the need for planetary defense posed by the asteroid/comet threat to mankind. Fusion rocket engines for planetary defense can satisfy a need that ignites the human imagination. Research in this area will address an immediate goal that will attract the next generation of researchers (and beyond) to our laboratories, universities and industry. We emphasize that we are not giving up on fusion's ultimate promise of clean abundant energy. Instead we have described an alternative goal: developing fusion rockets for high-speed comet intercept missions as part of a planetary defense program; which would generate a higher sense of urgency, and is of equal or greater importance to society.

\section{Summary}

A logical Planetary Defense Program would be interagency and international in nature. A Planetary Defense effort that can deal with both asteroids and comets requires several capabilities: early detection, good tracking and targeting, and early momentum change. Better (earlier) detection of potentially hazardous asteroids and comets is essential. If you can't see them in time, you can't put up a defense. In the near term we should practice deflection technologies on asteroids and short period comets, ideally ones that have no adverse consequence if the deflection doesn't match modeling. For the difficult comet intercept mission, we should begin now to develop high-specific power, high-specific impulse plasma rockets with fusion cores...because it is going to take a while. An intercept vehicle that has both a nuclear explosive payload and a nuclear rocket engine is necessary for the fast comet scenario. We emphasize that the best nuclear engine on the bottom of this rocket would be fusion powered, for performance that combines high specific power with high specific impulse, possibly reducing response times to a few months instead of the present value of years (or even "never" for comets).

The long-period comet threat will always be with us, whether or not an impact is next week, or thousands of 
years from now. Fusion energy is the critical science and technology needed to counter this threat.

Open Access This article is distributed under the terms of the Creative Commons Attribution 4.0 International License (http://crea tivecommons.org/licenses/by/4.0/), which permits unrestricted use, distribution, and reproduction in any medium, provided you give appropriate credit to the original author(s) and the source, provide a link to the Creative Commons license, and indicate if changes were made.

\section{References}

1. http://science.energy.gov/fes/. DOE Fusion Energy Sciences homepage

2. http://f4e.europa.eu/. European Fusion 4 Energy homepage

3. http://energy.gov/sites/prod/files/2014/04/f14/2014_dept_energy_ strategic_plan.pdf. US DOE Energy Strategic Plan 2014-2018, Ernest Moniz, DOE/CF-0067

4. http://science.energy.gov/ /media/fes/fesac/pdf/2003/Fesac_final_ non_elec_2003.pdf. Non Electric Applications of Fusion, Report to FESAC, July 31, 2003. See chapter V

5. S.K. Borowski, D.R. McCurdy, T.W. Packard, Nuclear thermal rocket/vehicle characteristics and sensitivity trades for NASA's Mars design reference architecture (DRA) 5.0 Study, in Proceedings of Nuclear and Emerging Technologies for Space 2009, paper 203599, Atlanta, GA, June 14-19 (2009)

6. P.J. Turchi, Propulsion Techniques, ISBN 1-56347-115-9 (American Institute of Aeronautics and Astronautics, 1998)

7. P.J. Turchi, Chapter 9, Electric rocket propulsion systems, in Space Propulsion Analysis and Design, ed. by R. Humble, G. Henry, W. Larson (McGraw-Hill, New York, 1995)

8. J. Cassibry, R. Cortez, M. Stanic, A. Watts, W. Seidler II, R. Adams, G. Statham, L. Fabisinski, Case and development path for fusion propulsion. J. Spacecr. Rockets 52(2), 595 (2015)

9. J.F. Santarius, Lunar He-3, fusion propulsion, and space development, April 5-7 1988, in The Second Conference on Lunar Bases and Space Activities of the 21st Century, vol. 1, ed. by W. W. Mendell. NASA Conference Publications 3166, p. 75 (1992)

10. N. Schulze, Fusion Energy for Space Missions in the 21st Century, NASA Technical Memorandum 4298 (1991)

11. J.F. Santarius, B.G. Logan, Generic magnetic fusion rocket model. J. Propuls. Power 14(4), 519 (1998)

12. C. Williams, AIAA Paper SP-108-2004, 2004. Application of Recommended Design Practices for Conceptual Nuclear Fusion Space Propulsion Systems, AIAA Paper 2004-3534 (2004)

13. E. Teller, A. Glass, T. Kennith Fowler, A. Hasegawa, J.F. Santarius, Space propulsion by fusion in a magnetic dipole. Fusion Sci. Technol. 22(1), 82 (1991)

14. J.F. Santarius, G.L. Kulcinski, L.A. El-Guebaly, G.A. Emmert, H. Khater, Z. Musicki, M.E. Sawan, I.N. Sviatoslavsky, W.F. Vogelsang, P.L. Walstrom, L.J. Wittenberg, Critical issues for SOAR: the space orbiting advanced fusion power reactor, January 1988 (revised August 1988). Presented at fifth symposium on space nuclear power systems, Albuquerque, NM, 11-14 Jan 1988; Space Nuclear Power Systems 1988 (Orbit, Malabar FL, 1989), p. 161

15. Y.S. Razin, G. Pajer, M. Breton, E. Ham, J. Mueller, M. Paluszek, A.H. Glasser, S.A. Cohen, A direct fusion drive for rocket propulsion. Acta Astronaut. 105, 145 (2014)

16. Y.C.F. Thio et al., High energy space propulsion based on magnetized target fusion. Paper AIAA 99-2703, 35th AIAA/
ASME/SAE/ASEE joint propulsion conference, Los Angeles, CA, 20-24 June (1999)

17. C.H. Williams, L.A. Dudzinski, S.K. Borowski, A.J. Juhasz, Glenn Research Center, Cleveland, Ohio, Realizing "2001: A Space Odyssey": Piloted Spherical Torus Nuclear Fusion Propulsion. NASA/TM-2005-213559, March 2005; for the 37th joint propulsion conference and exhibit, Salt Lake City, Utah, 8-11 July (2001)

18. C.D. Orth et al., The VISTA spacecraft-advantages of ICF for interplanetary fusion propulsion applications, in Proceedings of IEEE 12th Symposium on Fusion Engineering, vol. 2, p. 1017 (1987)

19. M. Paluszek, G. Pajer, Y. Razin, S.A. Cohen, J. Slonaker, M. Walsh, K. Griffin, R. Feder, Direct fusion drive for a human Mars orbital mission, in Proceedings of IAC, Toronto, (2014). Also, PPPL-5064 (2014)

20. P.J. Turchi, Fusion rocket based on stabilized liner implosions. IEEE Trans. Plasma Sci. Spec. Issue Plasma Propuls. 43(1), 240 (2015)

21. R.B. Adams, R. Alexander et al., Survey of technologies relevant to defense from near-earth objects, NASA/TP-2004-213089

22. Space Studies Board Aeronautics and Space Engineering Board, Division on Engineering and Physical Sciences National Research Council, National Academies, Defending Planet Earth: Near-Earth Object Surveys and Hazard Mitigation Strategies (The National Academies Press, Washington 2010)

23. J.B. Mueller, Y.S. Razin, M.A. Paluszek, A.J. Knutsen, G. Pajer, S.A. Cohen, A.H. Glasser, Direct fusion drive rocket for asteroid deflection, in Proceedings, IEPC-2013, Washington (2013)

24. M. Wall, Space.com, June 18, "Earth impact: are comets a bigger danger than asteroids? (2014), http://www.space.com/26264asteroids-comets-earth-impact-risks.html

25. Comet C/2013 A1 information, http://en.wikipedia.org/wiki/C/ 2013_A1; http://mars.nasa.gov/comets/sidingspring/

26. Table 1, NASA Office of Inspector General "NASA's Efforts to Identify Near-Earth Objects and Mitigate Hazards", IG-14-030, 15 Sept (2014)

27. J.L. Remo, The dilemma of nuclear energy in space. Bull. At. Sci. 71(3), 38 (2015)

28. NASA Near-Earth Object Survey and Deflection Analysis of Alternatives, Report to Congress (2007), http://neo.jpl.nasa.gov/ neo/report2007.html

29. J. Finseth, Overview of Rover engine test: final report, NASACR-184270 (1991), http://ntrs.nasa.gov/archive/nasa/casi.ntrs. nasa.gov/19920005899.pdf

30. D. Buden, Nuclear Thermal Propulsion Systems, Space Nuclear Propulsion and Power: Book 2, ISBN-978-0-9741443-3-7 (Polaris Books, 2011)

31. W.H. Robbins, H.B. Finger, An Historical Perspective of the NERVA Nuclear Rocket Engine Technology Program, NASA Contractor Report 187154/AIAA-91-3451, NASA Lewis Research Center, NASA (1991)

32. B.W. Longmier, M. Ballenger, C.S. Olsen, J.P. Squire, F.R. Chang Diaz, Performance studies of the VASIMR VX-200, AIAA-2011-1071, 49th AIAA Aerospace Sciences Meeting and Exhibit, Orlando, FL, 4-7 Jan (2011)

33. D.I. Poston, 2002, Nuclear design of SAFE-400 space fission reactor, Nuclear News (2001)

34. J.S. Sovey, V.K. Rawlin, M.J. Patterson, Ion propulsion development projects in US: space electric rocket test 1 to deep space 1. J. Propuls. Power 17(3), 517-526 (2001)

35. M. Rayman, T.C. Fraschetti, C.A. Raymond, C.T. Russell, Dawn: a mission in development for exploration of main belt asteroids Vesta and Ceres. Acta Astronaut. 58(11), 605-616 (2006)

36. E. Stuhlinger, Ion Propulsion for Space Flight (McGraw-Hill, New York, 1964) 
37. F. Dyson, Interstellar Transport, Phys. Today, p. 41-45 (1968)

38. Project Daedalus Study Group, A. Bond et al., Project Daedalus-The Final Report on the BIS Starship Study, JBIS Interstellar Studies, Supplement (1978)

39. REN21-Renewable Energy Policy Network for the 21 st Century Renewables 2012-Global Status Report (2012), http://www.
map.ren21.net/GSR/GSR2012.pdf; http://en.wikipedia.org/wiki/ Energy_development 